\title{
Climate model attractors: chaos, quasi-regularity and sensitivity to small perturbations of external forcing
}

\author{
V. P. Dymnikov and A. S. Gritsoun \\ Institute of Numerical Mathematics, Moscow, Russia \\ Received: 6 July 2000 - Revised: 25 October 2000 - Accepted: 28 November 2000
}

\begin{abstract}
In this paper we discuss some theoretical results obtained for climate models (theorems for the existence of global attractors and inertial manifolds, estimates of attractor dimension and Lyapunov exponents, symmetry property of Lyapunov spectrum). We define the conditions for "quasiregular behaviour" of a climate system. Under these conditions, the system behaviour is subject to the Kraichnan fluctuation-dissipation relation. This fact allows us to solve the problem of determining a system's sensitivity to small perturbations to an external forcing. The applicability of the above approach to the analysis of the climate system sensitivity is verified numerically with the example of the two-layer quasigeostrophic atmospheric model.
\end{abstract}

\section{Introduction}

One of the most important problems for present day science is the problem of prediction of climate changes due to human activity. All anthropogenic actions on the climate system (changes in greenhouse gas concentration, changes in the concentration of small gas fractions controlling the stratospheric ozone content, changes of albedo in the case of deforestation and so on) can be considered as small external perturbations. Consequently, this problem can be formulated as the problem of investigating the climate sensitivity to small external perturbations. The climate is assumed to be the ensemble of states that the climate system (the system consisting of atmosphere, hydrosphere, cryosphere, land surface and biota) passes through during a sufficiently long time period.

The formulated problem has one specific property: it leaves no room for a physical experiment. At the same time, because of specific features of the climate system there is also no room for laboratory experiments. The central method to investigate this problem is via numerical modelling. Since the only knowledge about the climate system that we have at

Correspondence to: A. S. Gritsoun (andrusha@inm.ras.ru) our disposal is the single trajectory of the system (from observational data), we must answer the fundamental question: what characteristics of the climate system should be reproduced by a numerical model in order that the sensitivity of the model be close to that of the climate system? To obtain the answer to this question (in the framework of some theory) we must make a series of assumptions about climate system behaviour. Note again that the problem of climate sensitivity dramatically differs from the problem of weather prediction, where we can use a statistical approach for model validation.

\section{2 "Ideal" climate model}

For further study we will suppose that there exists an "ideal model" of the climate system which has the following properties:

1. An "ideal model" of the climate system is a dissipative semi-dynamical system.

2. An "ideal model" has a global attractor.

3. The dynamics generated by the model is considered on the attractor of the model.

4. The trajectory of the model is unstable. The dynamics of the model is ergodic (the trajectory is dense on the model attractor) and chaotic (there are positive Lyapunov exponents). The trajectory can be regarded as a realization of certain ergodic random processes.

The idea of the construction of a mathematical climate theory (Dymnikov and Filatov, 1997) was to obtain constructive answers to the above question: to provide a constructive method of investigating the climate sensitivity to small external perturbations. 


\section{Climate model attractors: Lyapunov exponents and attractor dimension estimates}

Most modern climate models have the following form

$\frac{\partial \phi}{\partial t}+K(\phi) \cdot \phi=F(\phi, t)-D \phi,\left.\quad \phi\right|_{t=0}=\phi_{0}, \quad \phi \in \Phi,(1)$

where $\Phi$ is the phase space of the system (1) (real Hilbert space with scalar product (.,.)); $F$ is an external forcing; $D$ is a dissipative term, $(D \phi, \phi)>0 ; K(\phi)$ is a skew-symmetric operator depending linearly on the solution $\phi$

$(K(\phi) \cdot \phi, \phi)=0$.

Equation (2) is the condition for energy conservation in the absence of forcing and dissipation. In this case, the system has a number of additional conservation laws but they will not be mentioned below.

The hypotheses that were stated in the previous section of the paper mean that for the system (1) we must prove the theorems of global solvability and attractor existence. In addition, we must have an estimate of the attractor dimension and that of the Lyapunov exponents. In the general case, these theorems are not yet proven. However, some results for general climate models were obtained (Ladyzhenskaya, 1987; Lions et al., 1992, 1997). For less general models the situation is significantly better. As an example, we will cite the results obtained for the barotropic vorticity equation on the rotating sphere $S^{2}$

$$
\begin{aligned}
& \frac{\partial \Delta \psi}{\partial t}+J(\psi, \Delta \psi+f+H)=F-\alpha \Delta \psi+\mu \Delta^{2} \psi, \\
& \left.\psi\right|_{t=0}=\psi_{0},
\end{aligned}
$$

where $\psi$ is dimensionless streamfunction; $\mu, \alpha$ are friction coefficients; $F$ is an external forcing; $H$ is an orography; $f$ is the Coriolis parameter; $J(.,$.$) is the Jacobian, \Delta$ is the Laplacian operator.

This equation has a finite-dimensional global attractor $A$, whose fractal dimension $d_{F}$ can be estimated as follows (Ilyin, 1993; Dymnikov and Filatov, 1997)

$d_{F}(A) \leq\left(\frac{12}{\sqrt{\pi}}\right)^{2 / 3} G^{2 / 3}\left(\frac{1}{2}+\ln \frac{3 \sqrt{2}}{\sqrt{\pi}}+\ln G\right)^{1 / 3}$.

In this formula $G$ is the generalized Grashof number $(G=$ $\|F\|_{2} /\left(\mu\left|\lambda_{\max }\right|\right), \lambda_{\max }$ is the largest (negative) eigenvalue of the Laplacian operator $\left(\lambda_{\max }=-2\right.$ in the dimensionless form), $\|F\|_{2}=\left(\int_{S^{2}} F^{2} d s\right)^{1 / 2}, d s$ is the area element.

For the system (3) the existence of an inertial manifold (a smooth finite dimensional manifold that attracts every bounded set in the phase space of the system) was also proven and its dimension estimate was obtained. The consequence of these theorems is the fact that the infinite dimensional system (3) can be exactly parameterized by a smooth system on the inertial manifold.

\section{Finite dimensional approximation of the climate models}

Every climate model is in fact some spatial approximation of the original system of partial differential equations (1)

$\frac{d \phi^{h}}{d t}+K_{h}\left(\phi^{h}\right) \cdot \phi^{h}=F^{h}-D^{h} \phi^{h}$,
$\left.\phi^{h}\right|_{t=0}=\phi_{0}^{h}, \quad \phi^{h} \in \Phi^{h} \equiv R^{N}$.

The important problem here is the closeness of attractor characteristics between the original and approximating systems. Unfortunately up to now there has been no solution to this problem.

On the other hand, the finite dimensional model (5) itself can be considered as a climate model provided that it has conservation laws (in the absence of forcing and dissipation) similar to that of the original model (1). In particular, the following relationship must hold

$\left(K_{h}\left(\phi^{h}\right) \cdot \phi^{h}, \phi^{h}\right)_{N}=0$,

where $(., .)_{N}$ is a scalar product in $R^{N}$.

Hereafter we will assume that the approximating system (5) has all the important conservation laws inherent to the original partial differential system (under conditions of zero forcing and dissipation). For example, for the barotropic vorticity equation (3) it is important to conserve energy, enstrophy and vorticity for the correct reproduction of the spectral energy distribution. Generally, this problem (which conservation laws should be taken into account) is very difficult and will be discussed later (when the Lyapunov pairing property will be investigated). In the problem of trajectory prediction (for a finite period of time), the situation is much more evident. For the correct solution to this problem we have to prove only the solvability theorem for the original system and the theorem on convergence of the approximating system solution to that of the original system. In the climate prediction problem we have to approximate the attractor of the system and it is very important to take into account all aspects of the system dynamics.

For finite dimensional dissipative systems like (5), the proof of the global attractor existence theorem is very simple and is a consequence of the fact that the dissipative system in $R^{N}$ has a bounded, finite-dimensional absorbing set. The attractor dimension estimates can be obtained by means of calculation of the global Lyapunov exponents. The theoretical foundation for calculation of the Lyapunov exponents is the ergodic theorem of Oseledets (Oseledets, 1968).

Let

$Q^{h}=K_{h}\left(\phi^{h}\right) \cdot \phi^{h}+D^{h} \phi^{h}-F^{h}$.

Then the linearization of equation (5) with respect to the solution $\phi^{h}(t)$ can be written as

$\frac{d \phi^{\prime}}{d t}+A\left(\phi^{h}\right) \phi^{\prime}=0,\left.\quad \phi^{\prime}\right|_{t=0}=\phi_{0}^{\prime}$, 
and

$A \equiv \frac{\partial Q^{h}}{\partial \phi^{h}}$

is the Jacobi matrix.

Writing the system (6) in the resolved form

$\phi^{\prime}(t)=S(t) \phi_{0}^{\prime}$.

According to the Oseledets theorem the Lyapunov exponents $\sigma_{i}$ can be calculated by the formula

$\sigma_{i}=\lim _{t \rightarrow \infty} \frac{1}{t} \ln \lambda_{i}\left(S_{t}^{*} S_{t}\right), i=1 \ldots N$,

for almost all initial conditions $\phi_{0}^{h}$ with respect to the invariant ergodic measure of the system (5). In the formula (8), $\lambda_{i}\left(S_{t}^{*} S_{t}\right)$ is the $i$-th singular value of the operator $S_{t}$.

After the calculation of $\sigma_{i}$, one can estimate the fractal attractor dimension $d_{F}$ by applying the well known KaplanYorke formula (Kaplan and Yorke, 1979)

$d_{F}=J+\sum_{i=1}^{J} \sigma_{i} /\left|\sigma_{J+1}\right|$,

where the integer $J$ is determined as follows

$\sum_{i=1}^{J} \sigma_{i}>0, \quad \sum_{i=1}^{J+1} \sigma_{i}<0$,

and $\sigma_{i}$ 's are written in decreasing order.

The Lyapunov exponent distribution contains a lot of additional information about the system. The behaviour of the system will be chaotic if one or more Lyapunov exponents are positive. Hyperbolic systems have only one zero Lyapunov exponent (this exponent corresponds to the direction of motion). The attractor of these systems is structurally stable with respect to small external perturbation. Moreover, the response of these systems is linear with respect to the perturbations (if the perturbations are small enough) (Ruelle, 1998).

Consider the finite dimensional semi-dynamical system

$\frac{d \phi^{h}}{d t}+Q^{h}\left(\phi^{h}\right)=0,\left.\quad \phi^{h}\right|_{t=0}=\phi_{0}^{h}$.

Suppose that this system has an attractor $A$, so that if $\phi_{0}^{h} \in A$ then $\phi^{h}(t) \in A, \forall t>0$.

Apply a perturbation $\delta f^{h}$ to the right hand side of this system. The perturbed system will be of the form

$\frac{d \phi_{1}^{h}}{d t}+Q^{h}\left(\phi_{1}^{h}\right)=\delta f^{h},\left.\quad \phi_{1}^{h}\right|_{t=0}=\phi_{10}^{h}$

We will also assume that the perturbed system has its own attractor $A_{1}$ and $\phi_{1}^{h}(t) \in A_{1}, \forall t>0$ for all $\phi_{10}^{h} \in A_{1}$.

Let $\operatorname{dist}_{H}\left(A, A_{1}\right)$ be the Hausdorff distance between sets $A$ and $A_{1}$ :

$\operatorname{dist}_{H}\left(A, A_{1}\right)=\max \left\{\operatorname{dist}_{X}\left(A, A_{1}\right), \operatorname{dist}_{X}\left(A_{1}, A\right)\right\}$,
$\operatorname{dist}_{X}\left(A, A_{1}\right)=\sup _{a \in A} \inf _{a_{1} \in A_{1}} \rho\left(a, a_{1}\right)$,

where $\rho\left(a, a_{1}\right)$ is the distance between point $a$ and point $a_{1}$.

Kornev (1999) showed that, under certain conditions imposed on the system,

$\operatorname{dist}_{H}\left(A, A_{1}\right) \rightarrow 0$, if $\left\|\delta f^{h}\right\|_{2} \rightarrow 0$,

where $\left\|\delta f^{h}\right\|_{2} \equiv\left(\sum_{i}\left(\delta f_{i}^{h}\right)^{2}\right)^{1 / 2}, \delta f_{i}^{h}$ is the $i$-th component of $\delta f^{h}$.

Unfortunately, these conditions are not constructive in the sense that they can not be verified for most climate models.

\section{Sensitivity of attractors to the small perturbations}

Consider, once again, the finite dimensional system (5). Omit for simplicity the superscript $h$

$\frac{d \phi}{d t}+Q(\phi)=0,\left.\quad \phi\right|_{t=0}=\phi_{0}, \quad \phi \in R^{N}$.

Applying the small constant perturbation $\delta f$ to the right hand side of the system (10)

$\frac{d \phi_{1}}{d t}+Q\left(\phi_{1}\right)=\delta f,\left.\phi_{1}\right|_{t=0}=\phi_{10}$

Consider $\phi(t)^{\prime}=\phi_{1}(t)-\phi(t)$. From (10) and (11) it follows that

$\frac{d \phi^{\prime}}{d t}+Q_{\phi}^{\prime} \phi^{\prime}+R\left(\phi^{\prime}\right)=\delta f$

where $Q_{\phi}^{\prime}$ is the first derivative of $Q(\phi)$ at point $\phi(t)$ and $R\left(\phi^{\prime}\right)$ contains nonlinear terms with $\phi^{\prime}$.

Let $\delta \bar{\phi}$ be the response of the average state of the model (10) to the perturbation $\delta f$

$\delta \bar{\phi}=\left\langle\phi_{1}(t)\right\rangle_{1}-\langle\phi(t)\rangle$

$\left(\langle\ldots\rangle_{1}\right.$ and $\langle\ldots\rangle$ are averages over the equilibrium distributions of states for the perturbed and original systems respectively.)

The question we are interested in is, what is the response $\delta \bar{\phi}$ of the average state of the system to a small enough perturbation $\delta f$

$\delta \bar{\phi}=U(\delta f) ?$

Note that this question is a part of the more general problem of determining the stability of the invariant measure of the system (10) with respect to small constant perturbations. The properties of the response operator $U$ are not known in advance (for example, $U$ should be linear from the "physical" point of view but it also can be discontinuous - when a bifurcation occurs for some set of parameter values).

We can use the linear equation

$\frac{d \phi^{\prime}(t)}{d t}+Q_{\phi}^{\prime} \phi^{\prime}(t)=\delta f$

instead of (12) for infinitesimal $\delta f$ (provided that $Q(\phi)$ is smooth enough). 
The solution of (13) can be written as

$\phi^{\prime}(t)=\int_{0}^{t} K\left(t, t^{\prime}\right) d t^{\prime} \delta f$,

where $K\left(t, t^{\prime}\right)$ is the Green function of the linear system (13) $\left(K\left(t, t^{\prime}\right)=S(t) S^{-1}\left(t^{\prime}\right)\right.$, and $S(t) \phi_{0}^{\prime}$ is the solution of the system (13) with $\delta f=0$ and initial condition $\phi_{0}^{\prime}$ ).

If the invariant measure of the original system converges to the invariant measure of the perturbed system (after the perturbation has been applied) for some finite time $T$, we have $\left\langle\phi_{1}(t)\right\rangle(t)=\left\langle\phi_{1}(t)\right\rangle_{1}=\left\langle\phi_{1}\right\rangle_{1}$ for any $t>T$. Consequently, $\left\langle\phi^{\prime}(t)\right\rangle=\left\langle\phi_{1}\right\rangle_{1}-\langle\phi\rangle=\delta \bar{\phi}$, if $t>T$.

Applying the average $\langle\ldots\rangle$ to both sides of (14) and taking the integration time $t$ in (14) as $t \rightarrow \infty$, we get the linear response formula

$\delta \bar{\phi}=U \delta f, \quad U=\int_{0}^{\infty} K\left(t-t^{\prime}\right) d\left(t-t^{\prime}\right)$.

Recall that $\left\langle K\left(t, t^{\prime}\right)\right\rangle=K\left(t-t^{\prime}\right)$ due to the invariance of the measure.

Note that (15) is true only if the invariant measure of the original system is "strongly" stable with respect to infinitesimal $\delta f$.

For practical purposes, it is important to have a feasible method of investigation of system sensitivity (method for the construction of the response operator $U$ to small perturbations of external forcing). The systems for which this method could be constructed are the regular systems that will be considered in the next section of the paper.

\section{Regular systems: the Kraichnan theorem}

Consider equation (10). A regular system is one which has the quadratic conservation law $E$ and satisfies the Liouville equation for phase volume incompressibility. Namely, if

$2 E=\sum_{i=1}^{N} \alpha_{i} \phi_{i}^{2}=$ const, $\alpha_{i}>0$,

$\sum_{i=1}^{N} \frac{\partial Q_{i}}{\partial \phi_{i}}=0$

then the system (10) is called regular ( $\phi_{i}$ and $Q_{i}$ are the components of the solution $\phi$ and right hand side $Q$ respectively).

Let the system (10) be in equilibrium, that is, the initial distribution of points over system attractor $\rho(u)$ are the same at any moment in time. Due to (16) and (17), the equilibrium distribution will be the normal distribution

$\rho(u)=e^{-E}$.

The fluctuation-dissipation theorem (Kraichnan, 1959) relates the average impulse response operator $\left\langle K\left(t, t^{\prime}\right)\right\rangle$ of the equilibrium regular system to its statistics

$$
\left\langle K\left(t, t^{\prime}\right)\right\rangle=K\left(t-t^{\prime}\right)=C\left(t-t^{\prime}\right) C^{-1}(0) .
$$

In this equation $\langle\ldots\rangle$ is the average over the equilibrium distribution $\rho(u)$ and $C\left(t-t^{\prime}\right)=\left\langle u(t) u\left(t^{\prime}\right)^{T}\right\rangle$ is the autocorrelation matrix of the system (10) with lag $\left(t-t^{\prime}\right)$. (.. $)^{T}$ is the transpose operator.

Substituting relation (18) into the linear response formula (15) we finally arrive at the response formula (Leith, 1975)

$\delta \bar{\phi}=U \delta f=\int_{0}^{\infty} C(t) C^{-1}(0) d t \delta f$.

The importance of this equation is that, for ergodic systems, the response operator $U$ can be calculated from just a single trajectory (model data).

\section{Quasi-regular systems: Lyapunov pairing property}

Since the relationship (19) is not exact for the small but finite perturbation $\delta f$, an attractive idea is to use the formula (19) to approximate the response operators of dissipative systems. Of course, the forcing perturbations must be small enough and the dynamics of these systems must be "quasi-regular", that is:

- the contraction rate of the phase volume on a system attractor must be equal to zero,

- the system must have an approximate conservation law, - the equilibrium distribution of points on a system attractor must be close to the normal distribution.

Note that the energy of a dissipative system is finite and the average contraction rate of the phase volume on the attractor of the system is zero (this fact is used for the construction of attractor dimension estimates). Consequently, the fundamental question here is the meaning of the word "quasi". An even more important question is what assumptions must be made to ensure the applicability of the formula (19) for dissipative systems.

Consider this problem more precisely with the example of the Lyapunov exponents distribution. Let the system

$\frac{d \phi_{i}}{d t}+Q_{i}(\phi)=0, \quad i=1 \ldots N,\left.\quad \phi_{i}\right|_{t=0}=\phi_{i 0}$.

be the Hamiltonian system with time independent Hamiltonian $\mathcal{H}$. In this case, $\mathcal{H}$ is an integral of motion. It is well known that the distributions of global and local Lyapunov exponents of such a system are symmetrical with respect to zero. If the system (20) is not Hamiltonian but can be recast in Hamiltonian form with the help of some smooth mapping with a bounded smooth inverse, then the distribution of global Lyapunov exponents is again symmetrical (rather than that of the local exponents). This property of global Lyapunov exponents represents a set of additional conservation laws of the system.

Suppose, once again, that the system (20) can be recast in Hamiltonian form. If we add forcing $F$ and dissipation $-\alpha \phi$ $(\alpha>0)$ to the right hand side of the system (20) we obtain the dissipative system

$\frac{d \phi_{i}}{d t}+Q_{i}(\phi)=-\alpha \phi_{i}+F_{i}, i=1 \ldots N,\left.\phi_{i}\right|_{t=0}=u_{i 0}$. 


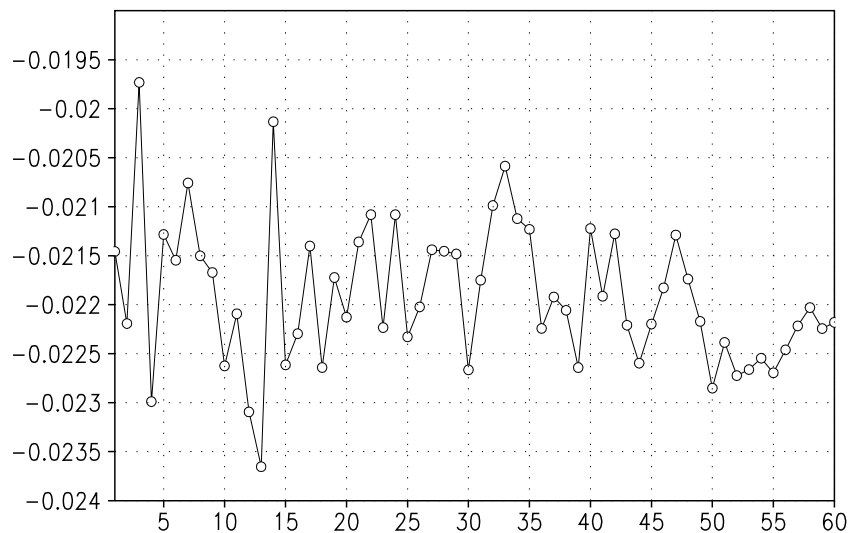

Fig. 1. The sum of global Lyapunov exponents $\lambda_{i}+\lambda_{N-i+1}$ for barotropic atmosphere model versus index $i$ (T15 truncation, $N=$ $120,2 \alpha=0.0227, \mu=0$, timestep $\Delta t=0.01$ ).

The Lyapunov exponents $\sigma_{i}$ of this system obey the following pairing property (Dymnikov and Gritsoun, 2001)

$\sigma_{i}+\sigma_{N+1-i}=-2 \alpha$.

(where $\sigma_{i}$ 's are taken in decreasing order). This result is an extension of the results of Dressler (1998). Note that such a form of Lyapunov exponents symmetry is a necessary condition for recasting the system in Hamiltonian form.

Now we investigate how this pairing property is satisfied for a finite-dimensional approximation of the barotropic vorticity equation (3) with $\mu=0$ (Dymnikov and Gritsoun, 2001)

$$
\begin{aligned}
& \frac{\partial \Delta \psi}{\partial t}+J(\psi, \Delta \psi+f+H)=F-\alpha \Delta \psi, \\
& \left.\psi\right|_{t=0}=\psi_{0} .
\end{aligned}
$$

More precisely, equation (22) is approximated with the help of a Galerkin method (where basic functions are the eigenfunctions of the Laplacian operator). We use two different truncations: T8 (the phase space dimension $N$ equals 36) and $\mathrm{T} 15(N=120)$. The Matsuno scheme is used as a timeintegration scheme

$\psi^{k+1}=\psi^{k}+\Delta t \mathcal{F}\left(\psi^{k}+\Delta t \mathcal{F}\left(\psi^{k}\right) / 2\right)$.

The value of the friction coefficient in the boundary layer $\alpha$ is the same in both experiments and corresponds to $1 / 14$ days $(\approx 0.01135$ in dimensionless form). We use the real orography $H$ in our model. Forcing $F$ is chosen so that the average state of the system is close to that of the January mean circulation on the $200 \mathrm{mb}$ surface.

The Lyapunov exponents of the models are calculated with the help of Oseledets theorem. Both these systems are chaotic (the model with resolution T8 has 12 positive exponents and the second one has 55). The sums of the $\sigma_{i}+\sigma_{N+1-i}$ are plotted in Fig. 1 (T15 truncation case) and Fig. 2 (T8 truncation case).

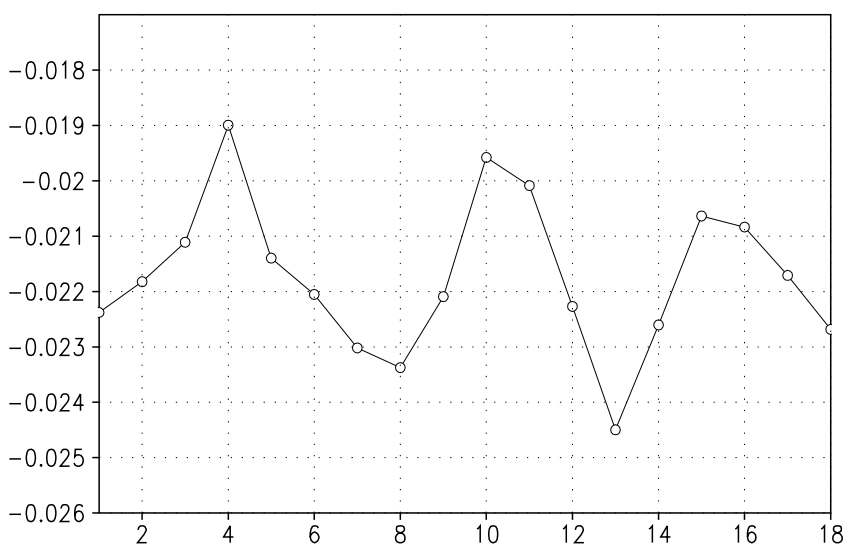

Fig. 2. The sum of global Lyapunov exponents $\lambda_{i}+\lambda_{N-i+1}$ for barotropic atmosphere model versus index $i$ (T8 truncation, $N=$ $36,2 \alpha=0.0227, \mu=0$, timestep $\Delta t=0.01$ ).

From these plots it is clear that both systems obey the pairing property of Lyapunov exponents fairly well. It should be pointed out that the rigorous theorem concerning the Hamiltonian representation for the space discretization of system (3) (in the absence of forcing and dissipation) is not obtained yet. However, the above numerical results are an indication of the fact that both systems are quasi-regular. At the same time, the Lyapunov pairing property is independent of the system dynamics and consequently can only be a marker of quasi-regularity of the system.

The natural requirement that ensures the quasi-regularity of the dissipative system dynamics on its attractor is the requirement for a large number of degrees of freedom (the "thermodynamic limit" requirement). In other words, the attractor dimension of the system must be large enough. Since all directions that correspond to the positive Lyapunov exponents fall onto the model attractor then the number of positive exponents also must be large. This means that the dynamics on the attractor of the system must be strongly chaotic. In this case, the distributions of system characteristics must be close to normal distributions due to the central limit theorem and the energy dispersion must be small compared with the energy itself.

\section{Numerical experiments (quasi-geostrophic two-layer model)}

In this part of the paper we will show how the fluctuationdissipation theorem can be used to predict the response of the two-layer baroclinic atmosphere model to small perturbations of external forcing.

The independent variables of the model are: $\psi_{l}, \psi_{u}$ the streamfunctions at the $750 \mathrm{mb}$ and $250 \mathrm{mb}$ levels respectively; $w_{500}$ the vertical p-velocity at $500 \mathrm{mb} ; \tau$ the temperature at $500 \mathrm{mb}$. (All variables and equations are in dimensionless form). 

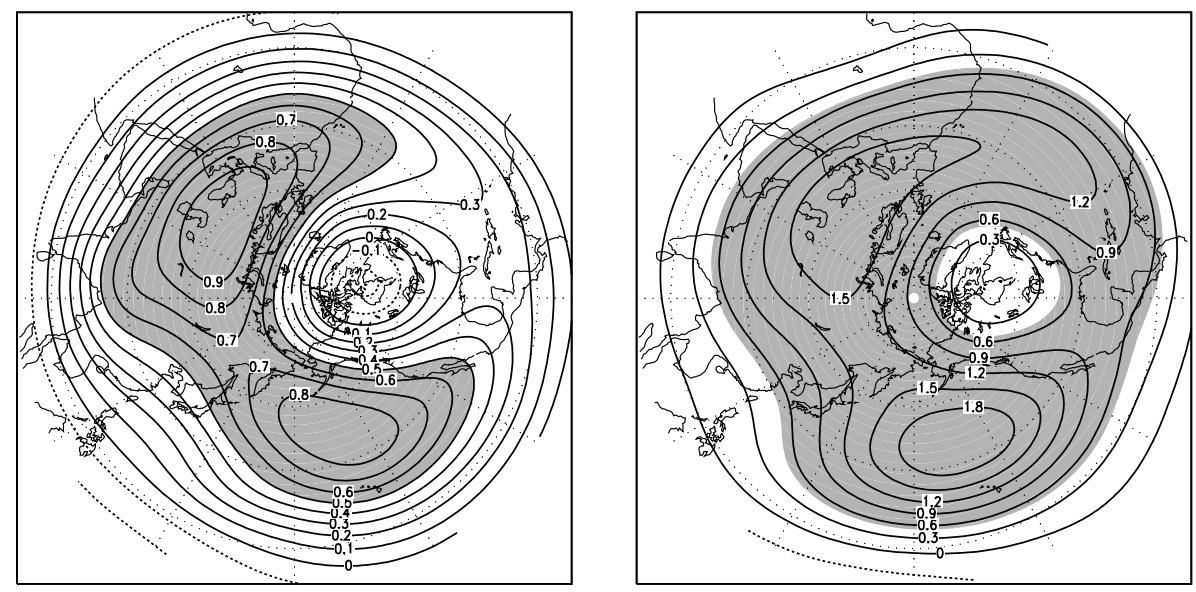

Fig. 3. The leading low-frequency EOF of the streamfunction field ( $41 \%$ of total dispersion, averaging time 30 days) in arbitrary units. Left plot corresponds to $750 \mathrm{mb}$ surface, right plot to $250 \mathrm{mb}$ surface.

1. Vorticity equations at the $750 \mathrm{mb}$ and $250 \mathrm{mb}$ surfaces:

$$
\begin{gathered}
\frac{\partial \Delta \psi_{l}}{\partial t}+J\left(\psi_{l}, \Delta \psi_{l}+f\right)=-A w_{500}+A w_{1000} \\
-\alpha \Delta \psi_{1000}+\alpha_{1} \Delta \psi_{500}+\mu \Delta^{2} \psi_{l}
\end{gathered}
$$

$$
\begin{gathered}
\frac{\partial \Delta \psi_{u}}{\partial t}+J\left(\psi_{u}, \Delta \psi_{u}+f\right)=A w_{500} \\
-\alpha_{1} \Delta \psi_{500}+\mu \Delta^{2} \psi_{l}
\end{gathered}
$$

2. Thermodynamic equation at the $500 \mathrm{mb}$ surface:

$\frac{\partial \tau}{\partial t}+J\left(\psi_{500}, \tau\right)=\sigma^{2} w_{500}+\alpha_{s}\left(\tau_{s f}-\tau\right)+\mu \Delta \tau$

3. The balance equation at the $500 \mathrm{mb}$ surface:

$$
A^{\prime}\left(\psi_{l}-\psi_{u}\right)=-2 \delta^{2} \tau
$$

Dependent variables are: the stream function at 500 and 1000 $\mathrm{mb}$, and the vertical $p$-velocity at $1000 \mathrm{mb}$ :

$\psi_{500}=0.5\left(\psi_{l}+\psi_{u}\right), \psi_{1000}=1.5 \psi_{l}-0.5 \psi_{u}$,

$w_{1000}=-2 k J\left(\psi_{1000}, H\right)$.

In the above equations: $f$ is the Coriolis parameter, $\Delta$ the Laplacian operator, $\nabla$ the gradient operator, $\nabla \cdot$ the divergence, $A x=\nabla \cdot\left(f \nabla \Delta^{-1} x\right), A^{\prime} x=\Delta^{-1} \nabla \cdot(f \nabla x)$.

Forcing and parameters: $\tau_{s f}$ the surface temperature, $H$ the orography, $\delta^{2}=R T_{0} /\left(2 \Omega^{2} L^{2}\right), \sigma^{2}=N_{B V}^{2} R T_{0} / g^{2}, k=$ $\rho g H_{0} /(2 \Delta p), N_{B V}$ Brunt-Väisälä frequency, $\Omega$ the Earth's angular velocity, $L$ the Earth's radius, $R$ the gas constant, $H_{0}=1000 \mathrm{~m}, g=10 \mathrm{~m} / \mathrm{s}^{2}, \rho=1 \mathrm{~g} / \mathrm{cm}^{3}, T_{0}=250 \mathrm{~K}$, $\Delta p=500 \mathrm{mb}, \alpha=\alpha_{s}=1 / 7$ days, $\alpha_{1}=1 / 60$ days, $\mu=$ $8.0 \cdot 10^{-5}$.

The system (23-26) can be reduced to the following system of two equations

$$
\begin{aligned}
& \frac{\partial \Delta \psi_{s}}{\partial t}+J\left(\psi_{s}, \Delta \psi_{s}+f\right)+J\left(\psi_{a}, \Delta \psi_{a}\right)= \\
& -k A J\left(\psi_{s}+2 \psi_{a}, h\right)-\alpha \Delta \psi_{s} / 2-\alpha \Delta \psi_{a}+\mu \Delta^{2} \psi_{s},
\end{aligned}
$$

$$
\begin{aligned}
& \frac{\partial(\Delta-\gamma B) \psi_{a}}{\partial t}+J\left(\psi_{a}, \Delta \psi_{s}+f\right)+J\left(\psi_{s}, \Delta \psi_{a}\right)= \\
& -k A J\left(\psi_{s}+2 \psi_{a}, h\right)+\gamma A J\left(\psi_{s}, A^{\prime} \psi_{a}\right)-\alpha \Delta \psi_{s} / 2-\alpha \Delta \psi_{a} \\
& +\alpha_{1} \Delta \psi_{s}+\mu \Delta^{2} \psi_{a}+\gamma \alpha_{s} A\left(\delta^{2} t_{s f}+A^{\prime} \psi_{a}\right)-\mu \gamma A \Delta A^{\prime} \psi_{a}, \\
& \text { where } \psi_{s}=\left(\psi_{l}+\psi_{u}\right) / 2, \psi_{a}=\left(\psi_{l}-\psi_{u}\right) / 2, \gamma=1 /\left(\sigma^{2} \delta^{2}\right) \\
& =\left(L / L_{R}\right)^{2}, L_{R}^{2}=N_{B V}^{2} R^{2} T_{0}^{2} /\left(2 \Omega^{2} g^{2}\right), L_{R}=800 \mathrm{~km}, \\
& \gamma=120, B=A A^{\prime} .
\end{aligned}
$$

For the spatial approximation of the system the Galerkin method is used (for which the basis functions are the eigenfunctions of the Laplacian operator; we use triangular truncation T21; the phase space dimension $=2 \times 440=880$ ). The Matsuno scheme is chosen as the time integration method.

For this model we calculate the prediction $U$ of the model response operator with the help of the formula (19). To that end we perform a 30000-days model run. Using this data we calculate the average state of the model, its EOFs, and its low-frequency EOFs. As an example, the first low-frequency EOF of the model is presented in Fig. 3. The first picture corresponds to the $750 \mathrm{mb}$ surface, the second to the $250 \mathrm{mb}$ surface.

Because of a numerical instability in the calculation of $C(0)^{-1}$ (due to an insufficient amount of data), the perturbations $\delta f$ are considered in the subspace of the first 30 EOFs of the system and thus the response operator matrix has the form

$U=\int_{0}^{\infty} C(t) d t V D_{30}^{-1} V_{30}^{T}$

In this formula: $C(0)^{-1}=V D^{-1} V^{T}, V_{30}=\left\{v_{1}, \ldots, v_{30}\right.$, $0, \ldots, 0\}, D_{30}^{-1}=\operatorname{diag}\left\{d_{1}^{-1}, \ldots, d_{30}^{-1}, 0, \ldots, 0\right\} . d_{i}$ is the dispersion that corresponds to the $i$-th EOF of the system $v_{i}$.

We calculate the singular value decomposition (SVD) of $U$

$U=Q \Lambda P^{T}$,

where $\Lambda=\left\{\lambda_{1}, \ldots, \lambda_{30}, 0, \ldots, 0\right\}, P=\left\{p_{1}, \ldots, p_{30}\right\}, Q=$ $\left\{q_{1}, . ., q_{30}\right\}$.

Recall that

$\sup _{x \in R^{N}} \frac{\|U x\|_{2}}{\|x\|_{2}}=\lambda_{1}$, 

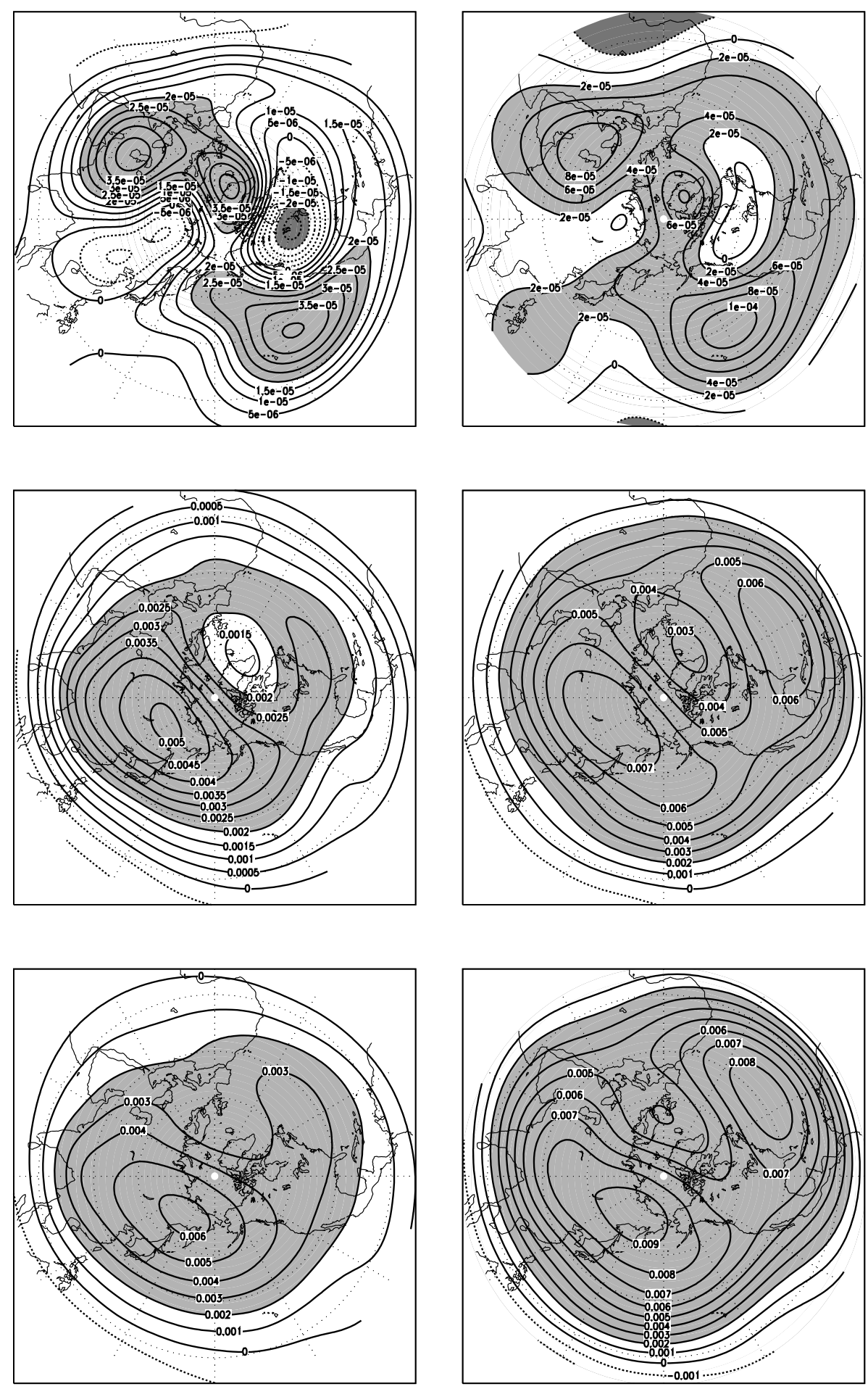

Fig. 4. The first right singular vector $p_{1}$ of $U$ in arbitrary units. Left plot corresponds to $750 \mathrm{mb}$ surface, right plot to $250 \mathrm{mb}$ surface.

Fig. 5. Prediction $\epsilon \lambda_{1} q_{1}\left(\epsilon \lambda_{1} q_{1}=\right.$ $\left.\epsilon U p_{1}\right)$ of the model response to forcing perturbation $\epsilon p_{1}$ (arbitrary units).

Fig. 6. Real model response to forcing perturbation $\epsilon p_{1}$. and the maximum is attained on the first right singular vector $p_{1}$ that corresponds to $\lambda_{1}$. Moreover, $U p_{1}=\lambda_{1} q_{1}$. Consequently, the right singular vector $p_{1}$ of $U$ can be naturally called an optimal perturbation of the system and the vector $\lambda_{1} q_{1}$ (that is proportional to the first left singular vector $q_{1}$ of $U)$ its maximum response.

For the first two optimal perturbations $p_{1}$ and $p_{2}\left(p_{2}\right.$ is the second right singular vector of $U$ ), the responses of the model are calculated directly. For this purpose, the perturbations $\delta f=\epsilon p_{1}$ and $\delta f=\epsilon p_{2}(\epsilon$ is a small normalizing constant) were added into the right hand side of the model and two 10000-day integrations were performed to calculate new average states of the system. The model responses obtained (differences between new and old averege states of the system) were then compared with the predicted responses $\epsilon \lambda_{1} q_{1}$ and $\epsilon \lambda_{2} q_{2}$. 

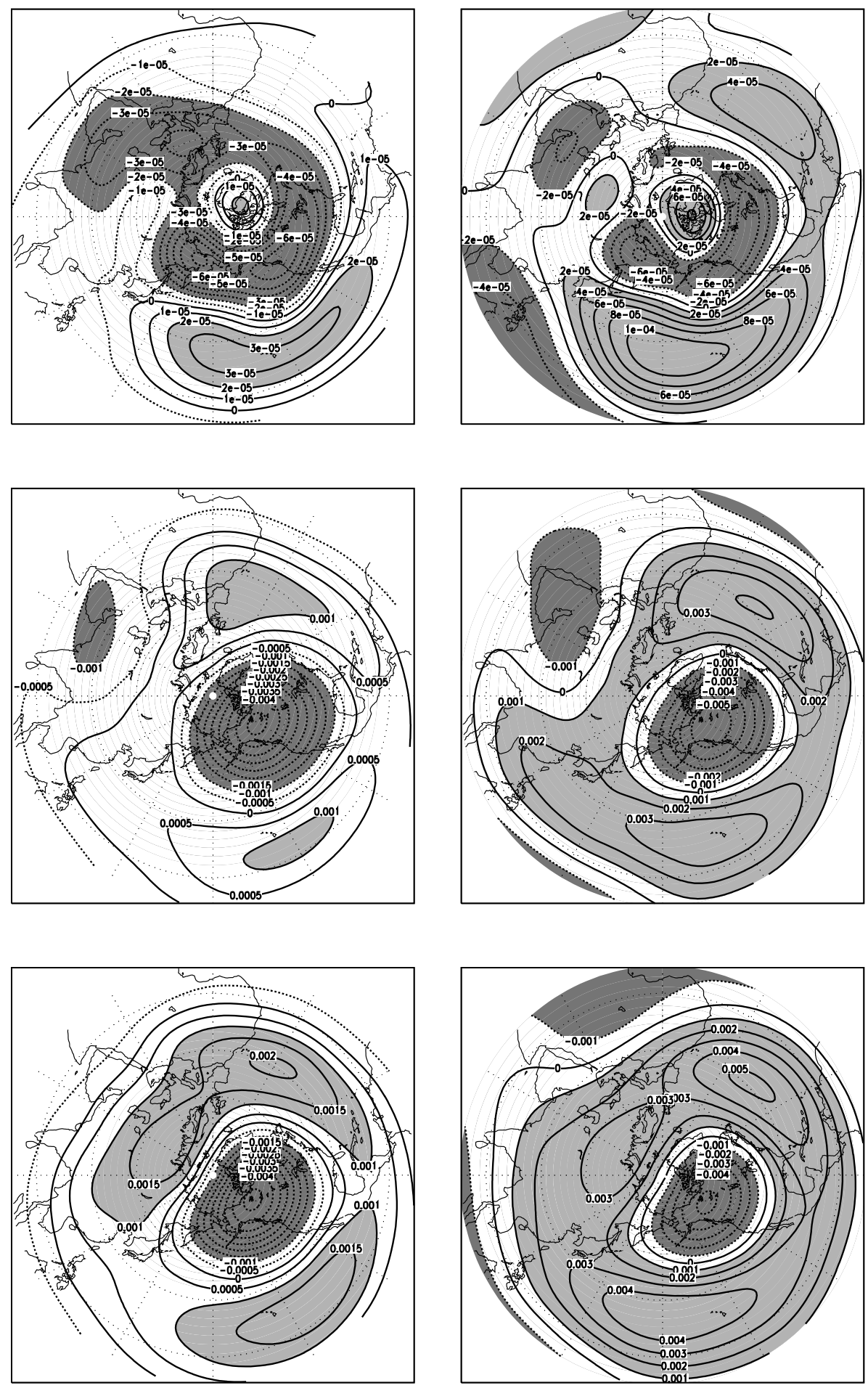

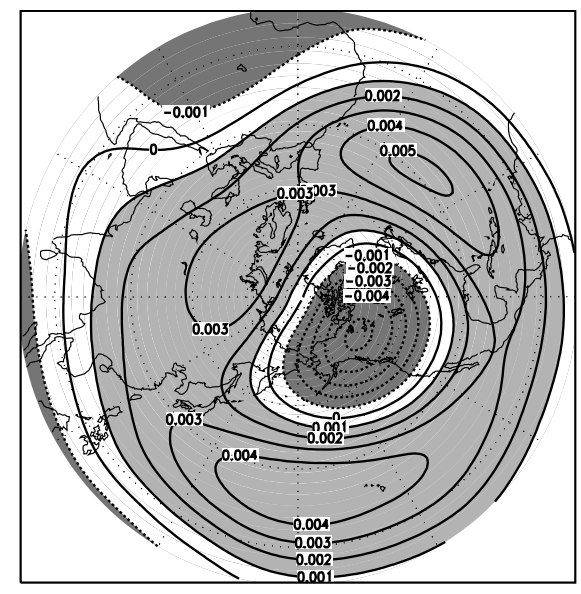

Fig. 7. The second right singular vector $p_{2}$ of $U$ in arbitrary units. Left plot corresponds to $750 \mathrm{mb}$ surface, right plot to $250 \mathrm{mb}$ surface.

Fig. 8. Prediction $\epsilon \lambda_{2} q_{2}\left(\epsilon \lambda_{2} q_{2}=\right.$ $\left.\epsilon U p_{2}\right)$ of the model response to forcing perturbation $\epsilon p_{2}$ (arbitrary units).

Fig. 9. Real model response to forcing perturbation $\epsilon p_{2}$.
The first optimal perturbation $p_{1}$ is presented in Fig. 4 (again the first picture corresponds to the $750 \mathrm{mb}$ surface, the second to the $250 \mathrm{mb}$ surface). The predicted response $\epsilon \lambda_{1} q_{1}$ to this perturbation is shown in Fig. 5, and the corresponding model response in Fig. 6 . The correlation between actual and predicted model responses (between Figs. 5 and 6) is 0.99 . The real amplification coefficient of the norm of the perturbations is in good agreement with the predicted ampli- fication coefficients $\lambda_{1}$ (151 and 125 respectively).

The results of the second numerical experiment (with $\delta f=$ $\left.\epsilon p_{2}\right)$ are presented in Figs. 7-9. Again, either the direction of the actual model response or its magnitude is close to the predictions $q_{2}$ and $\lambda_{2} \epsilon$.

The evident conclusion is that the fluctuation-dissipation theorem provides a very good prediction of the response of our quasigeostrophic atmospheric model to small constant 
perturbations of external forcing.

\section{Conclusions}

In this paper we discuss the problem of predicting of the climate system sensitivity to small external perturbations. By imposing conditions of "quasiregularity" on the system dynamics, we obtain a method of construction for analysing its sensitivity which is based on the Kraichnan fluctuationdissipation theorem. The essence of these conditions is the requirement that the system must have a large number of degrees of freedom.

It is shown that the above approach provides an effective prediction method for the response of the quasigeostrophic atmospheric model to small external perturbations. This conclusion is consistent with the results that were obtained previously for a barotropic atmospheric model by Dymnikov and Gritsoun (1999).

Acknowledgements. This work is supported by the Russian Foundation for Basic Research (grant N99-05-64038) and French-Russian A. M. Lyapunov Institute (grant N4-97).

\section{References}

Dressler, U., Symmetry property of the Lyapunov spectra of a class of dissipative systems with viscous damping, Phys. Review A, 38, N4, 2103-2109, 1998.

Dymnikov, V. and Filatov, A., Mathematics of climate modelling, Birkhäuser, Boston, 1997.

Dymnikov, V. P. and Gritsoun A. S., Lyapunov exponent pairing property on the climate model attractors, Izvestia RAS, Atmo- spheric and Oceanic Phys., 36, N3, 2001 (in press).

Gritsoun, A. and Dymnikov, V., Barotropic atmosphere response to small external actions. Theory and numerical experiments, Izvestia RAS, Atmospheric and Oceanic Phys., 35, N5, 511-525, 1999.

Ilyin, A. A., On the dimension of attractors for Navier-Stokes equations on two-dimensional compact manifolds, diff. and integral equat., 6, 183-214, 1993.

Kaplan J. L., and Yorke J. A., Chaotic behaviour in multidimensional difference equations, Lecture Notes in Math., 730, 228, 1979

Kornev, A. A., One criterion for complete continuity of attractors with respect to a parameter for some class of semidynamic systems, Doklady Rus. Acad. Nauk., 369, N5, 597-599 (Doklady Mathematics, 60, N3, 403-405), 1999.

Kraichnan, R. H., Classical fluctuation-relaxation theorem, Phys. Rev., 113, 1181-1182, 1959.

Ladyzhenskaya, O. A., On the determination of minimal global attractors for the Navier-Stokes and other partial differential equations, Uspekhi Mat. Nauk, 42, N6, 25-60, (Russian Math. Surveys, 42, N6, 25-60), 1987.

Leith, C. E., Climate response and fluctuation dissipation, J. Atmos. Sci., 32, 2022-2026, 1975.

Lions, J. L., Temam, R., and Wang, S., New formalities of the primitive equations of atmosphere and applications, Nonlinearity, 5 , 237-288, 1992.

Lions, J. L., Manley, O. P., Temam, R., and Wang, S., Physical interpretation of the attractor dimension for primitive equations of atmosphere circulation, J. Atmos. Sci., 54, 1137-1143, 1997.

Oseledets, V. I., A Multiplicative ergodic theorem. Lyapunov characteristic numbers for dynamical systems, Trudy Mosk. Mat. Obsc., 19, 179-210 (Moscow Math. Soc., 19, 197-210), 1968.

Ruelle, D., General linear response formula in statistical mechanics and the fluctuation-dissipation theorem far from equilibrium, Phys. Letters A, 245, 220-224, 1998. 